\title{
Evaluating Modularization Quality as an Extra Objective in Multiobjective Software Module Clustering
}

\author{
Márcio de Oliveira Barros \\ Post-graduate Information Systems Program - PPGI/UNIRIO \\ Av. Pasteur 458, Urca - Rio de Janeiro, RJ - Brazil \\ marcio.barros@uniriotec.br
}

\begin{abstract}
The application of multiobjective optimization to address Software Engineering problems is a growing trend. Multiobjective algorithms provide a balance between the ability of the computer to search a large solution space for valuable solutions and the capacity of the human decision-maker to select an alternative when two or more incomparable objectives are presented. However, when more than a single objective is available to be taken into account in a search process, the number of objectives to be considered becomes part of the decision. We have examined the effectiveness of using modularization quality (MQ) as an objective function in the context of the software module clustering problem. We designed and executed a set of experiments using both randomlygenerated and real-world instances of varying size and complexity and a fixed calculation budget set in a per instance basis. Results collected from these experiments show that using MQ as an extra objective can improve search results for small instances (few modules to be clustered), while it decreases search quality for larger instances (more than 100 modules to be clustered). Search quality was measure both in terms of the number of distinct solutions found and on their coverage of the solution space, according to the spread and hypervolume quality indicators. We correlated problem characteristics (number of modules, clusters, and dependencies), instance attributes (module dependency distribution patterns), and algorithmic measures (MQ conflict with cohesion and coupling) and found that these elements can only partially explain the effectiveness of using MQ as an extra objective.
\end{abstract}

Keywords: multiobjective optimization, software clustering, coupling, cohesion, genetic algorithms.

Acknowledgements. The author would like to express his gratitude for FAPERJ and $\mathrm{CNPq}$, the research agencies that financially supported this project. 